\title{
Guía para la evaluación de la Usabilidad en los Entornos Virtuales de Aprendizaje (EVA)
}

Andrea C. Alarcón-Aldana(1), Edilma L. Díaz ${ }^{(1,2)}$ y Mauro Callejas-Cuervo(1)

(1) Grupo de Investigación en Software GIS, Facultad de Ingeniería, Universidad Pedagógica y Tecnológica de Colombia, Avenida Central del Norte 39-115, Sede Central Tunja-Boyacá-Colombia (e-mail: andrea.alarconaldana@uptc.edu.co,edilmaleonordiaz@hotmail.com,maurocallejas@gmail.com) (2) Facultad de Ingeniería de Sistemas, Fundación Universitaria Juan de Castellanos, Carrera 11 No.11-44, Tunja, Colombia (e-mail: edilmaleonordiaz@hotmail.com)

Recibido Dic. 16, 2013; Aceptado Ene. 16, 2014; Versión final recibida Feb. 12, 2014

\section{Resumen}

Se presenta el diseño y construcción de una guía para la evaluación de usabilidad en entornos virtuales de aprendizaje, a partir de la identificación de algunos criterios que permiten cuantificar el grado de satisfacción de los usuarios de este tipo de herramientas; para ello se llevó a cabo el desarrollo de las siguientes fases: identificación de atributos de usabilidad, caracterización de los entornos virtuales de aprendizaje, estructuración de criterios y métricas de la guía, y finalmente la validación de la guía propuesta, para lo cual se evaluaron dos entornos virtuales de aprendizaje, uno de uso libre y otro propietario, demostrando que la guía propuesta es un instrumento que puede ser aplicado por diversos tipos de usuarios para verificar el grado de usabilidad de dichas aplicaciones.

Palabras clave: usabilidad, entorno virtual de aprendizaje, guía de evaluación, interfaz de usuario, calidad de software.

\section{Guidelines for Assessing Virtual Learning Environments (VLE) Usability}

\begin{abstract}
This work presents the design and construction of the guidelines for usability assessment in virtual learning environments, based on some criteria identification, which allow to quantify the satisfaction rate of users of this type of tools. For this purpose, the following stages were developed: identification of usability attributes, virtual learning environment characterization, guidelines' criteria and metrics structuring and finally, validation of the proposed guidelines. To do so, two virtual learning environments were assessed: one of free use and the other one of proprietary use. It was shown that the proposed guidelines area tool that can be applied by different types of users to verify the usability degree of such applications.
\end{abstract}

Keywords: usability, virtual learning environment, assessment guidelines, user interface, software quality. 


\section{INTRODUCCIÓN}

El proceso enseñanza aprendizaje cuenta con diversas herramientas y metodologías que permiten la transmisión de conocimientos, entre las que se menciona e-learning, dejando de ser simplemente, depósitos de un curso en un computador; sino que también se han de considerar los diversos recursos de apoyo que facilitarán el proceso de enseñanza y en especial el soporte informático o plataforma sobre la cual se implantará; por tal razón, los Entornos Virtuales de Aprendizaje (EVA) se convierten en uno de los factores determinantes para la gestión de conocimiento, de esta manera estas herramientas permiten abordar nuevos escenarios y propenden por la calidad de la educación (Barragán y Ruíz, 2013), (Casas y Stojanovic, 2013); otros autores han definido modelos, técnicas y metodologías, que fomentan e institucionalizan la virtualización de la educación en todos los niveles y áreas (Monguillot, Guitert y González, 2013), (González y Esteban, 2013), pero es necesario que estas herramientas presenten características propias para facilitar el aprendizaje, surge entonces la necesidad de abordar la necesidad como uno de los principales criterios que se han de estudiar al momento de implementarlas.

La usabilidad, definida por González y Farnós (2009) como "la medida del grado de facilidad en el uso de un tipo de producto (en este caso "tecnológico") y del tipo de satisfacción que genera ese uso en el usuario", denotando que un buen producto software además de ser comprensible, debe satisfacer las necesidades y provocar interés del usuario sobre la aplicación; ahora bien en el marco de eLearning, la usabilidad con mayor razón, está ligada a la opinión del usuario, ya que éste persigue la apropiación de conocimientos y es fundamental que la interfaz gráfica sea lo suficientemente amigable para la consecución de dicho propósito.

Diversos autores y expertos en el tema, a través de sus investigaciones han comprobado que la usabilidad es uno de los factores críticos al momento de garantizar el éxito o fracaso de cualquier sitio y aplicación informática (Hurtado, 2013; Perurena, 2004), en especial de los EVA por ser el principal recurso de apoyo con que se cuenta para la formación académica a distancia, evidenciando la necesidad de unificar dichos criterios, ratificando la necesidad de estudiarlos en detalle y relacionarlos para así poder proponer una guía de evaluación de la usabilidad en la cual se integre y facilite el proceso de evaluación de usabilidad en Entornos Virtuales de Aprendizaje, tomando como fundamento los criterios de usabilidad propuestos por diversas normas (ISO 9126, 1991), modelos (Alfonzo, 2011; Pérez, 2007), estándares y técnicas orientadas a evaluar la usabilidad (Sánchez, 2011; Claros y Collazos, 2009) a partir de la experiencia tomada como fundamento en la investigación (Sánchez, 2003; Szpiniak y Sanzen, 2009). De igual forma es importante tener en cuenta que un factor clave para medir la usabilidad es la identificación de las características implícitas y explícitas de la interfaz de usuario de una aplicación informática (Herrera 2014, Tabares 2013), dado que el éxito o fracaso de un producto de este tipo, está altamente ligado a la satisfacción y comodidad percibida por el usuario final.

Ahora bien, antes de establecer el EVA a implantar dentro de una institución educativa, es recomendable llevar a cabo un análisis previo de los productos existentes en el mercado, con el fin de establecer el que se ajusta más a las necesidades particulares, y así crear un marco de trabajo definido y claro a sus actores, quienes han de participar activamente en sus procesos, apoyando las estrategias y metas de la institución. A partir de esta estrategia de trabajo se pueden incluir soluciones libres o propietarias, con el fin de establecer la posibilidad que tiene una institución para desarrollar sus actividades académicas, en procura de velar por la calidad del software (León, 2013) y ofrecer una herramienta que permita identificar la afinidad de una herramienta con las necesidades de un cliente/usuario en particular, se ha diseñado la Guía de evaluación de Usabilidad de Entornos Virtuales de Aprendizaje, la cual pretende determinar el grado de usabilidad en este tipo de aplicaciones.

El documento se presenta en cuatro secciones principales, iniciando con la descripción del diseño experimental que guía el desarrollo de la investigación, en seguida se explica el modelo de cuantificación de usabilidad en entornos virtuales de aprendizaje, especificando los subcriterios de cada uno de los criterios abordados, para posteriormente en la sección de resultados presentar la ponderación de los criterios, la escala de medición propuesta y los resultados de la aplicación de la guía en la evaluación de usabilidad de dos entornos virtuales de aprendizaje, por último se expone las conclusiones obtenidas.

\section{DESARROLLO DE LA INVESTIGACIÓN}

La investigación propuesta se llevó a cabo de acuerdo a unas etapas definidas las cuales se presentan en la Figura 1, donde se identifica que para el diseño de la guía fue necesario estudiar modelos, normas y/o estándares que permitieran recopilar los ítems de Usabilidad en las aplicaciones web, posteriormente se define las características propias de los EVA, para poder conocer las funcionalidades y objetivos de este tipo de aplicaciones enfocado a establecer los criterios, ítems y métricas necesarias para que por medio de una guía se obtenga un valor numérico que coadyuve a conocer las características de usabilidad de un EVA 
específico, finalmente se llevó a cabo el proceso de validación de la guía de evaluación propuesta, la cual fue aplicada a dos EVA (uno de tipo propietario, y otro de software libre), por estudiantes y docentes que hacen uso de estas herramientas en su proceso de formación, a quienes se les entregó la guía de evaluación que comprende unas instrucciones de uso, así como los criterios de usabilidad establecidos y un manual de usuario a través del cual se dan a conocer las pautas a tener en cuenta al momento de diligenciarla. Es de mencionar que las primeras etapas del diseño experimental abordado en la investigación están fuera del alcance de este artículo, el cual se enfoca a la descripción de la guía diseñada y la validación de la misma.

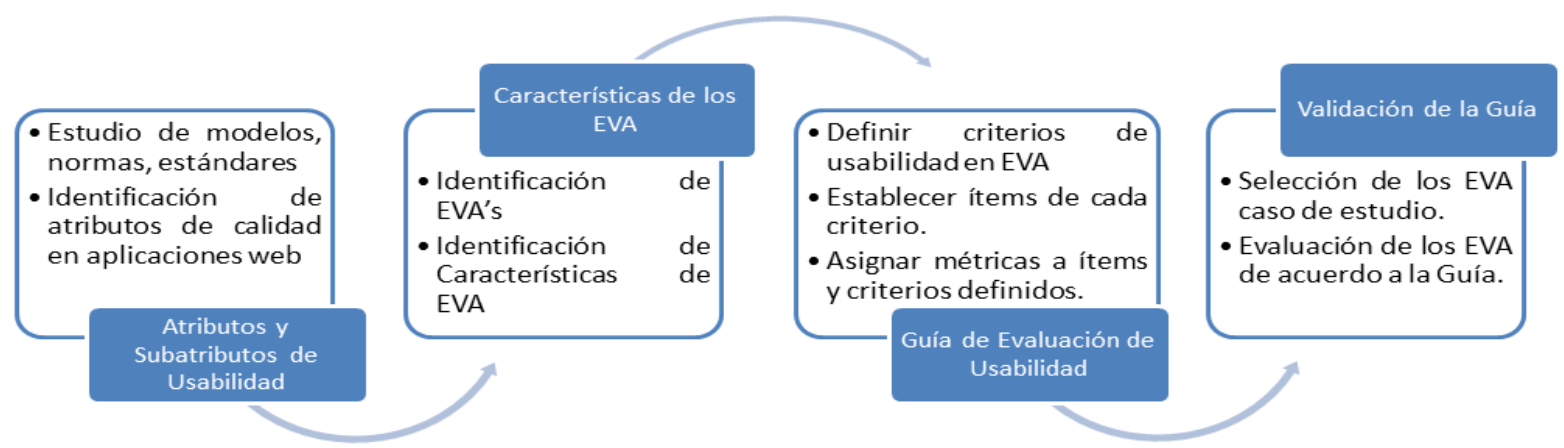

Fig. 1. Diseño experimental de la investigación

\section{MODELO DE CUANTIFICACIÓN DE LA USABILIDAD}

En el marco del desarrollo de la guía propuesta se establece el modelo que permite cuantificar los criterios para determinar el grado de usabilidad de los EVA, este modelo es denominado "criterio de Calificación de la Usabilidad de Entornos Virtuales de Aprendizaje -CUEVA-", el cual da a conocer el grado de usabilidad del EVA evaluado, teniendo en cuenta principalmente lo establecido por la ISO/9126 "Grado en que el software hace óptimo el uso de los recursos del sistema" (ISO 9126, 1991). El modelo es el resultado de la sumatoria de las evaluaciones de cada uno de los ocho criterios de usabilidad tenidos en cuenta, a saber: el Criterio de Comunicación (CC), el Criterio de Factores Estéticos (CFE), el Criterio de Operatividad (CO), el Criterio de Facilidad de Uso (CFU), el Criterio de Aprendizaje y factores Humanos (CA), el Criterio de Facilidad de Comprensión (CFC), el Criterio de Entrenamiento (CE) y el Criterio de Documentación (CD); la ecuación 1 muestra el valor de usabilidad de un EVA.

$\mathrm{CUEVA}=\mathrm{CC}+\mathrm{CFE}+\mathrm{CO}+\mathrm{CFU}+\mathrm{CA}+\mathrm{CFC}+\mathrm{CE}+\mathrm{CD}$

Donde el valor de usabilidad según CUEVA debe ser mayor que 0,0 y menor o igual a 5,0, correspondiente al rango de evaluación.

A cada criterio de usabilidad, se le asigna una ponderación (entre 0 y 100) o peso porcentual, ponderación que a su vez es distribuida entre los subcriterios establecidos para el análisis de las mismas, de acuerdo a la prioridad que posee cada criterio en el factor Usabilidad de un EVA.

Cada criterio que hace parte del modelo CUEVA, esta subdividido en subcriterios que facilitan la evaluación de usabilidad, destacando que los elementos en mención hacen referencia a las características propias de la herramienta más no a los contenidos de ésta, sin embargo, de acuerdo a la percepción del usuario en algunos casos podría afectar su valoración. A continuación se describe cada uno de los criterios y subcriterios contemplados.

\section{Criterio Comunicación (CC)}

El indicador de esta sección, mide el grado de Comunicación, o grado de interacción entre el software y el usuario, indicando las facilidades a nivel de recursos y medios que ofrece el entorno para interactuar con el usuario final; este criterio se subdivide en los siguientes subcriterios: Control sobre los medios (csm), Integración (i), Adecuación (a), Densidad (d), Compatibilidad con Diferentes Clientes Web (ccdcw), Presentación de los Archivos de Descarga (pad), Independencia de la resolución de la Pantalla (irp), Tecnologías auxiliares (ta), Idiomas (id); a los cuales se le asigna una valoración promedio de acuerdo a la calificación que oscila entre 0,0 y 5,0 dada por el evaluador y un peso porcentual de acuerdo a la ponderación asignada al criterio. El indicador está dado por: $C C=c s m+i+a+d+c c d c w+p a d+i r p+t a+$ id. 


\section{Criterio Factores Estéticos (CFE)}

El indicador de esta sección, mide los Factores Estéticos de la aplicación Web, es decir el entorno artístico de la interfaz gráfica de usuario, la cual se subdivide en el subciterio Estética (es) al cuales se le asigna una valoración de acuerdo a la calificación promedio que oscila entre 0 y 5 dada por el evaluador y un peso porcentual de acuerdo a la ponderación asignada al criterio. El indicador está dado por CFE = es.

\section{Criterio Operatividad (CO)}

Esta sección, cuantifica el grado de Operatividad es decir la capacidad del sitio Web para cumplir las funciones establecidas por el usuario, la cual se subdivide en los siguientes subcriterios: Mecanismo de Cancelación (mc), Mecanismo de gestión (mg), Acciones Mínimas (am), Proporción de Nombres Adecuados para los enlaces (pnae), Proporción de Elementos que muestran el estado actual (pemea), Utilización de Servicios de Contenido (usc), Satisfacción (sa) ; a los cuales se le asigna una valoración promedio de acuerdo a la calificación que oscila entre 0 y 5 dada por el evaluador y una ponderación o peso porcentual de acuerdo a la ponderación asignada al criterio. El indicador está dado por: $\mathrm{CO}=\mathrm{mc}+\mathrm{mg}+\mathrm{am}+\mathrm{pnae}+$ pemea + usc + sa

\section{Criterio facilidad de Uso (CFU)}

Esta sección, cuantifica la Facilidad de Uso es decir el grado de satisfacción del usuario frente al producto, la cual se subdivide en los siguientes subcriterios: Control (c), Flexibilidad de la Utilización de los Servicios (fus), Controles de Navegación (cn), Estado del Sistema (es), Menús (m), Control de Avance y Regreso Lógico (carl), Enlaces (e) ; a los cuales se le asigna una valoración promedio de acuerdo a la calificación que oscila entre 0 y 5 dada por el evaluador y un peso porcentual de acuerdo a la ponderación asignada al criterio . El indicador está dado por: $\mathrm{CFU}=\mathrm{c}+\mathrm{fus}+\mathrm{cn}+\mathrm{es}+\mathrm{m}+\mathrm{carl}+\mathrm{e}$.

\section{Criterio Aprendizaje - Factores Humanos (CA)}

Esta sección, cuantifica el Aprendizaje y Factores Humanos en la cual se establece de qué manera los usuarios evalúan e interactúan con el producto a través del grado de asimilación de conocimientos; esta sección se subdivide en los siguientes subcriterios: Familiar (f), Consistencia (c), Lenguaje Común (Ic), Intuitivo (i), predecible (pr), Conocimiento (cn) ; a los cuales se le asigna una valoración promedio de acuerdo a la calificación que oscila entre 0 y 5 dada por el evaluador y un o peso porcentual de acuerdo a la ponderación asignada al criterio. El indicador está dado por: $\mathrm{CA}=\mathrm{f}+\mathrm{c}+\mathrm{lc}+\mathrm{i}+\mathrm{pr}+\mathrm{cn}$

\section{Criterio Facilidad de Comprensión (CFC)}

Esta sección, mide la Facilidad de Comprensión, es decir la capacidad que ofrece el sitio para usar sus recursos en tareas y condiciones de uso particular, la cual se subdivide en los siguientes subcriterios: Tabla de Contenido (tc), Estructura del Sitio (esi), Facilidad de Reconocer (fr), Claridad en la Definición de requerimientos de Entrada/Salida (cdres), Técnicas de Redacción de Contenido Apropiado (trca), Comunicación Funcional (cf), Preclasificación de Contenidos (pc), Utilización de Formatos (uf), Fiable (fi), Definición de Perfiles de Usuario (dpu), Definición de Objetos del Sitio Discriminados por Perfiles (dodp); a los cuales se le asigna una valoración porcentual de acuerdo a la calificación que oscila entre 0 y 5 dada por el evaluador y un peso porcentual de acuerdo a la ponderación asignada al criterio. El indicador está dado por: $\mathrm{CFC}=\mathrm{tc}+\mathrm{esi}+\mathrm{fr}+\mathrm{cdres}+\operatorname{trca}+\mathrm{cf}+\mathrm{pc}+\mathrm{uf}+\mathrm{fi}+\mathrm{dpu}+\mathrm{dodp}$

\section{Criterio Entrenamiento (CE)}

Esta sección, mide el Entrenamiento o capacidad que ofrece el sitio para usar sus recursos para obtener conocimientos, desarrollar habilidades y capacidades al interactuar con este, la cual se subdivide en los siguientes subcriterios: Estrategias para Facilitar la Recordación (efr), Mecanismos de Anotación y Bitácoras de Usuario (mabui), Mecanismos de Señalamiento de Pasos dentro de una tarea (mspdt), Mecanismos para Suspender y Retomar Tareas (msrt), Información útil y Contextualizada (iuc), Mecanismos de Realimentación (mr), Mecanismos de Participación (mp), Ayuda de Búsqueda (ab); a los cuales se le asigna una valoración promedio de acuerdo a la calificación que oscila entre 0 y 5 dada por el evaluador y un peso porcentual de acuerdo a la ponderación asignada al criterio . El indicador está dado por: $\mathrm{CE}=\mathrm{efr}+$ mabui + $m s p d t+m s r t+i u c+m r+m p+a b$

\section{Criterio Documentación (CD)}

Esta sección, mide la incidencia de la Documentación es decir, la capacidad que ofrece el sitio al proveer documentos que faciliten la interacción con el sitio Web, la cual se subdivide en los siguientes subcriterios: Documentación (d), Relación Densidad/Utilidad (rdu), mecanismos de Acceso y Disposición (mad); a los 
cuales se le asigna una valoración promedio de acuerdo a la calificación que oscila entre 0 y 5 dada por el evaluador y un peso porcentual de acuerdo a la ponderación asignada al criterio. El indicador está dado por: $C D=d+r d u+$ mad.

Ahora bien, luego de identificar los criterios y subcriterios que hacen parte del modelo, la ecuación 2 muestra que la calificación de cada subcriterio se obtiene a partir del producto entre la valoración que registra el evaluador (entre 0,0 y 5,0) y la ponderación interna asignada; posteriormente se obtiene la cuantificación para cada criterio a partir del resultado entre la sumatoria de las calificaciones de cada subcriterio, multiplicada por el valor de ponderación de criterio (tabla 1), como se muestra en la ecuación 3.

subcritertio $=$ valoración evaluador * ponderación interna de subcriterio

critertio $=\sum($ subcriterios $){ }^{*}$ ponderación

Finalmente al llevar a cabo la sumatoria de los ocho criterios, se obtiene el valor de CUEVA, dada entre 0,0 y 5,0, que es realmente la calificación otorgada a la usabilidad de un entorno virtual de aprendizaje, de acuerdo a la percepción de un evaluador específico.

\section{RESULTADOS}

En la guía de evaluación de usabilidad, se tuvo en cuenta los ocho criterios descritos y que son recopilación de las metodologías y modelos que evalúan calidad de software web, tratados por autores como: Sicilia y García (2003), Gomes y Faria (2012), entre otros. A cada criterio se le asignó la respectiva ponderación según la prioridad de acuerdo a las características, metas y propósitos de un EVA (Álvarez, Avello y López, 2013) (Mestre, U., Fonseca y Valdés, 2007) como se muestra en la Tabla 1.

Tabla 1. Ponderación Factores de Usabilidad en los Entornos Virtuales de Aprendizaje (EVA)

\begin{tabular}{|l|c|c|}
\hline \multicolumn{1}{|c|}{ Factor } & Abreviatura & Ponderación \\
\hline Criterios comunicación y comprensibilidad & CC & $12,0 \%$ \\
\hline Criterio facilidad estética & CFE & $12,5 \%$ \\
\hline Criterio operatividad & CO & $13,5 \%$ \\
\hline Criterio facilidad de uso & CFU & $15,0 \%$ \\
\hline Criterio aprendizaje - factores humanos & CA & $12,5 \%$ \\
\hline Criterio facilidad de comprensión & CFC & $12,5 \%$ \\
\hline Criterio entrenamiento & CE & $11,5 \%$ \\
\hline Criterio documentación & CD & $10,5 \%$ \\
\hline
\end{tabular}

Con el fin de guiar al evaluador, y contar con una herramienta que emita resultados confiables, se establece una escala de juicio de valor que puede tener cada criterio, como se muestra en la tabla 2.

Tabla 2. Cuantificación de criterios de Juicio de Valor

\begin{tabular}{|l|c|}
\hline \multicolumn{1}{|c|}{ Criterio de juicio de valor } & Valor \\
\hline Se cumple plenamente. & 5,0 \\
\hline Se cumple en alto grado. & 4,0 a 4,9 \\
\hline Se cumple aceptablemente. & 3,0 a 3,9 \\
\hline Se cumple insatisfactoriamente. & 1,0 a 2,9 \\
\hline No se cumple. & 0,0 a 0,9 \\
\hline
\end{tabular}

Contando con la información descrita en apartados anteriores, se procede a la estructuración de la guía de evaluación de usabilidad de EVA, la cual se ha presentado de forma preliminar en una hoja de cálculo, en la cual inicialmente se informa la función de cada pestaña que aparece en la parte inferior de la hoja, pestañas que identifican los criterios de usabilidad establecidos a través del estudio y que se evaluaran mediante la plantilla. De igual manera, en el documento se da a conocer la escala de las métricas para cada ítem, la cual se ha determinado en el rango de cero $(0,0)$ a cinco $(5,0)$, donde cero $(0,0)$ indica que no se satisface el criterio evaluado y cinco $(5,0)$ que cumple en su totalidad. Así mismo, se presenta cada una de los formularios asociadas a los ocho criterios descritos, en los que se presenta los subcriterios, y se solicita al evaluador determinar el valor para cada uno de ellos; finalmente se dan a conocer los resultados obtenidos de la evaluación en el formulario de resultados, en el cual se visualiza un resultado cuantitativo sobre el grado de Usabilidad del EVA evaluado, a partir de la ecuación CUEVA. Parte de la información y estructura de la guía propuesta se muestra en la Figura 2. 


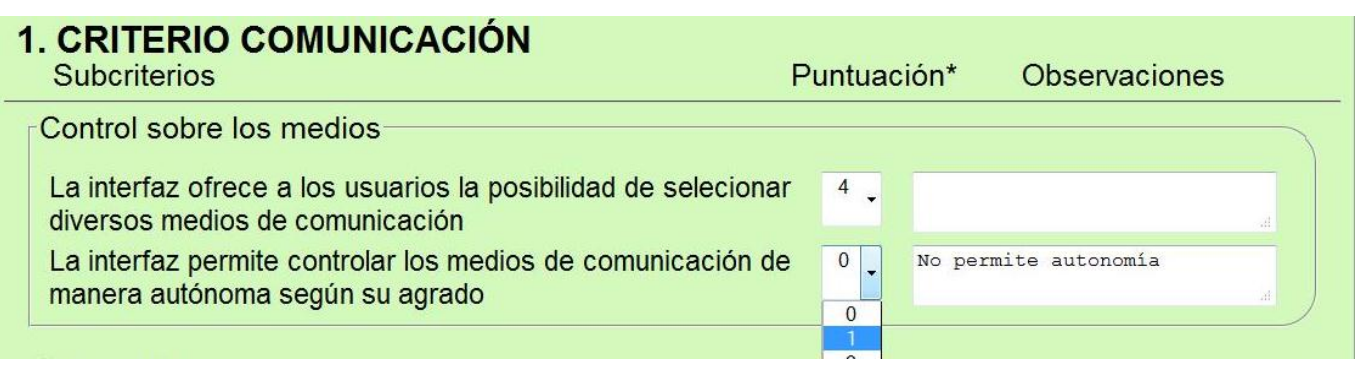

Fig. 2. Criterio comunicación en la guía para la evaluación de la Usabilidad en los EVA.

Para la validación de la guía de evaluación de usabilidad se seleccionaron dos entornos virtuales de aprendizaje como caso de estudio, uno de tipo software libre y el segundo software propietario; la muestra usada para la aplicación de la evaluación fue de ochenta (80) personas, entre los cuales se agrupan profesores y estudiantes que utilizan dichas herramientas en Instituciones de Educación Superior, con el propósito de conocer la validez y aplicabilidad de la guía y también determinar el grado de usabilidad de las herramientas seleccionadas, obteniendo así los siguientes resultados.

Con respecto a la evaluación de usabilidad del EVA de tipo libre y siguiendo el instrumento propuesto, se obtiene que la calificación fue de 3,2 (en un rango de cero a cinco), como se describe en la tabla 3. La explicación de los valores obtenidos en la evaluación de cada uno de los criterios para el caso de estudio EVA libre, se presenta a continuación.

Tabla 3. Cuantificación Criterios de Usabilidad de las Plataforma Evaluadas

\begin{tabular}{|l|c|c|}
\hline \multicolumn{1}{|c|}{ Criterio } & $\begin{array}{c}\text { Valoración } \\
\text { Plataforma Libre }\end{array}$ & $\begin{array}{c}\text { Valoración Plataforma } \\
\text { Propietaria }\end{array}$ \\
\hline Comunicación & 3,4 & 3,9 \\
\hline Facilidad Estética & 3,4 & 3,9 \\
\hline Operatividad & 3,0 & 4,0 \\
\hline Facilidad de uso & 3,1 & 4,0 \\
\hline Aprendizaje y Factores humanos & 3,5 & 4,0 \\
\hline Facilidad de Comprensión & 3,6 & 4,0 \\
\hline Entrenamiento & 3,1 & 4,0 \\
\hline Documentación & 2,0 & 3,7 \\
\hline
\end{tabular}

Criterio de Comunicación: según los datos obtenidos a través de la guía se ha demostrado que en el EVA libre evaluado. Este criterio se cumple aceptablemente ya que los recursos con que cuenta no son adecuados, dificultando la interacción del usuario con el sitio, así como el logro de los objetivos que persigue la herramienta.

Criterio Facilidad Estética: los resultados indican que este criterio se cumple aceptablemente ya que su contenido y estructura no se encuentran bien balanceados, teniendo en cuenta los recursos estéticos necesarios para presentar al usuario un ambiente armonioso y coherente, lo cual al no ser de esta manera, dificulta su funcionalidad.

Criterio Operatividad: este criterio se cumple aceptablemente ya que los mecanismos presentados, y acciones sobre los mismos no son suficientes, ni adecuados, limitando la interacción con la herramienta, haciendo rutinario el trabajo del usuario.

Criterio Facilidad de Uso: en cuanto a este criterio, se evidencia que cumple aceptablemente dado que los servicios, recursos y entorno de la aplicación, aunque cumplen con las funcionalidades básicas, no son eficientes teniendo en cuenta el tipo de usuarios, diversidad de los mismos y servicios que ha de ofrecer.

Criterios Aprendizaje y factores humanos: se obtiene que este criterio se cumple aceptablemente debido a que los recursos con que cuenta la herramienta, no contribuyen de manera efectiva a la adquisición de conocimientos al no permitir adaptarse a las diferentes formas de aprendizaje de los estudiantes.

Criterio Facilidad de Comprensión: este criterio se cumple aceptablemente expresando que los recursos ofrecen una mediana claridad con respecto a los servicios que presta al momento de realizar las tareas, con mayor razón en condiciones particulares. 
Criterio Entrenamiento: de igual manera, este criterio se cumple aceptablemente pues los medios que ofrece no son suficientes para capacitar respecto al manejo del sitio, conllevando a que sea el usuario quien se autocapacite en cuanto a las características del sitio y los servicios que ofrece.

Criterio Documentación: La aplicación cuenta con escasa documentación que permita al usuario conocer e interactuar de manera apropiada con el sitio, lo cual hace que los usuarios perciban que este criterio se cumple aceptablemente.

Ahora bien, con respecto al EVA de tipo propietario, de igual manera en la tabla 3 se aprecia que los usuarios otorgaron un valor de cuatro cero 4,0 a la usabilidad de la aplicación; así, de acuerdo a la evaluación dada por los usuarios, se puede mencionar lo siguiente para cada uno de los criterios.

Criterio de Comunicación: los recursos con que cuenta no son adecuados dificultado su interacción con el usuario, por lo tanto se considera que cumple aceptablemente con este criterio.

Criterio Facilidad Estética: de igual manera que el criterio anterior, éste se cumple aceptablemente dado que su contenido y estructura no son los apropiados para considerarse un ambiente armonioso y coherente, dificultando su funcionalidad.

Criterio Operatividad: la aplicación evaluada cumple en alto grado con este criterio, en virtud a que los mecanismos y acciones son suficientes y adecuados, contribuyendo a facilitar la interacción con la herramienta, y motivando al usuario para que aproveche al máximo sus recursos.

Criterio Facilidad de Uso: los servicios, recursos y entorno de la herramienta se consideran eficientes, teniendo en cuenta el tipo de usuarios y servicios ofrecidos, demostrando que este criterio se cumple en alto grado.

Criterios Aprendizaje y factores humanos: los recursos con que cuenta la aplicación, contribuyen de manera efectiva a la adquisición de conocimientos, por lo cual se considera que este criterio se cumple en un alto grado.

Criterio Facilidad de comprensión: este criterio se cumple en un alto grado, expresando que los recursos son claros y coherentes respecto a los servicios que presta al momento de realizar las tareas en condiciones particulares.

Criterio Entrenamiento: este criterio se cumple en un alto grado en razón a que los medios que ofrece son suficientes para capacitar al usuario respecto al manejo del sitio, teniendo en cuenta las características del sitio, los servicios que ofrece y los roles de usuario que pueden acceder a estos.

Criterio Documentación: la aplicación cuenta con escasa documentación que permita al usuario conocer e interactuar de manera apropiada con el sitio, conllevando a que se considere que este criterio se cumple aceptablemente.

En términos generales el EVA propietario tomado como caso de estudio, muestra un alto grado de cumplimiento de usabilidad (4,0 según la guía de evaluación), evidenciando que ha sido diseñado específicamente para desarrollar la tarea de apoyar los procesos de formación a nivel de la educación superior, teniendo en cuenta las características, necesidades y diversidad de usuarios a los que está dirigida la herramienta.

Finalmente, a partir de la prueba de validación se determina que la guía propuesta es una herramienta que puede ser aplicada por diversos tipos de usuarios para verificar el grado de usabilidad de un EVA, independientemente de la filosofía o licenciamiento que ofrezca la herramienta. Además ofrece un resultado cuantitativo que permite al evaluador y/o usuario conocer el grado de satisfacción en el uso de este tipo de herramientas, al igual que posibilita a usuarios con roles directivos tomar decisiones frente a la selección del EVA apropiado para una comunidad académica.

\section{CONCLUSIONES}

La Usabilidad de una aplicación web es un criterio importante en el rendimiento y calidad del producto software, y aunque su evaluación puede tener un componente subjetivo, el estudio de normas, guías, estándares y diferentes propuestas permite unificar los aspectos relevantes en este proceso y servir como base para estructurar una guía, como la desarrollada en esta investigación, con el propósito de apoyar la evaluación de usabilidad, en este caso de Entornos Virtuales de Aprendizaje. 
Los resultados arrojados por el ejercicio de validación de la guía, mostraron que la herramienta de software propietario, frente a la plataforma de software libre, presenta una mayor calificación en el grado de usabilidad percibida por los usuarios que intervinieron en el proceso, sin afirmar que se pueda generalizar estos resultados a todas las plataformas existentes en el mercado.

Al finalizar la investigación se deduce que la guía propuesta es un instrumento válido para apoyar y propender por la calidad del software, debido a que permitió identificar los criterios de usabilidad que debe cumplir un EVA, y cuantificar la opinión de los usuarios, quienes son los que finalmente se benefician de que un producto software presente las características funcionales y de usabilidad que se espera.

Como trabajo futuro se propone la creación de una aplicación web que sistematice la guía presentada y evitar la manipulación de valores en los criterios tenidos en cuenta, como también facilitar el trabajo del evaluador y poder presentar los resultados de una manera más amigable.

\section{REFERENCIAS}

Alfonzo P., Revisión de modelos de calidad orientados a sitios web bancarios: Estudio preliminar, Rev. Técnica administrativa, 10(4), 1-10, (2011).

Álvarez, H., Avello, R., López, R., Los entornos virtuales de aprendizaje como recurso didáctico en el ámbito universitario. Rev. Universidad y Sociedad, 5(1), 1-10, (2013).

Barragán, R. y Ruiz E., Brecha de género e inclusión digital. El potencial de las redes sociales en educación, Rev. Profesorado, (en línea), 71(1), 309-323, (2013).

Pizarro, H.L., N.N. Mercado y J.L. Castillo, Análisis de Datos Experimentales de Lubricantes, Int. J. Lubrication, 22(4), 123-131 (2003).

Casas, M. y Stojanovic, L., Innovación en la universidad iberoamericana. Rev. Universidad y Sociedad del Conocimiento, 10(1), 61-74, (2013).

Claros, I. y C. Collazos, Propuesta Metodológica para la evaluación de la Usabilidad en Sitios Web: Experiencia Colombiana, Actas del VII Congreso Internacional de Interacción Persona-Ordenador, Universidad de Castilla, La Mancha, España 13 al 17 de Noviembre (2006).

Gomes, W., Faria, R. Calidad de los sitios web en la percepción de los usuarios. Rev. Estudios y perspectivas en turismo, (en línea), 21(4), 923-944, (2012).

González, A., Farnós, J., Usabilidad y accesibilidad para un e-learning inclusivo, Rev. Educación Inclusiva, 2(1), 49-60, (2009).

González, K. y Esteban, C., Caracterización de modelos pedagógicos en formación e-learning, Rev. Virtual Universidad Católica del Norte, (en línea), 39(3), 4-16, (2013).

Herrera, E. y Fernández, L., Diseño de objetos gráficos-interactivos, Rev. Icono 14, (en línea), 12(1), 219243, (2014).

Hurtado, L., Castrillón, O. y Olivar, G., Una Metodología Automatizada para la Evaluación de Usabilidad de Interfaces de Supervisión Industrial. Información Tecnológica, (en línea), 24(4), 95-104, (2013).

ISO 9126: Software product evaluation - Quality characteristics and guidelines for their use. (1991).

León, Y., Enrique, Góngora, A., Febles, A., Aplicando métricas de calidad a proyectos y procesos durante las pruebas exploratorias. Rev. Cubana de Ciencias Informáticas, (en línea), 7(2), 36-48, (2013).

Mestre, U., Fonseca, J., y Valdés, P., Entornos virtuales de enseñanza aprendizaje, (en línea), 1a edición, El Vedado, Ciudad de La Habana, Editorial Universitaria, (2007).

Monguillot, M., Guitert, M., González, C., El trabajo colaborativo virtual: herramienta de formación del profesorado de educación física. Rev. Retos. Nuevas tendencias en Educación Física, Deporte y Recreación, 24(2), 24-27, (2013). 
Pérez I., M., Construcción y Validación del cuestionario: Métrica de Calidad de Credibilidad e Interacción de Cursos de Teleformación. Rev. Iberoamericana de Tecnología en Educación y Educación en Tecnología, 2 , 30-38, (2007).

Perurena, L., y Moraguez, M., Usabilidad de los sitios Web, los métodos y las técnicas para la evaluación. Rev. Cubana de Información en Ciencias de la Salud, 24(2), 1-19, (2004).

Sánchez, J.; Una metodología para desarrollar y evaluar la usabilidad de entornos virtuales basados en audio para el aprendizaje y la cognición de usuarios ciegos. Rev. Iberoamericana de Educación a Distancia, 13(2), 265-293, (2010).

Sánchez, W.; Compendio de estándares, métodos, técnicas y buenas prácticas de ingeniería de la usabilidad orientado a sitios web en El Salvador. Actas del Concapan XXXI, Universidad Don Bosco, El Salvador Noviembre (2011).

Sicilia, M.A. y García, E. On the concepts of usability and reusability of learning objects. Rev. International Review of Research in Open and Distance Learning. (en línea), 4(2), 1-11, (2003).

Szpiniak, A. y C. Sanzen; Hacia un Modelo de Evaluación de Entornos Virtuales de Enseñanza y Aprendizaje (EVEA). Rev. Iberoamericana de Tecnología en Educación y Educación en Tecnología, (en línea), 4, 10-21, (2009).

Tabares, Angel U., Socarrás, Leoder A. Sistema de construcción de interfaces gráficas de usuario en entornos 3D con OGRE. Rev. Serie Científica, (en línea), 6(8), 1-11, (2013). 
